\title{
UNA DE CAL POR LAS QUE VAN DE ARENA: UN ESTUDIO DIACRÓNICO DE LOS ESTUCOS DE CALAKMUL Y PALENQUE
}

\author{
Isabel. Villaseñor y James Aimers
}

\begin{abstract}
RESUmEN: Este artículo presenta los resultados de los análisis de petrografía y fluorescencia de rayos $\mathrm{X}$ de materiales constructivos de Calakmul y Palenque. Las diferencias observadas en las muestras reflejan distintas tradiciones constructivas y los cambios tecnológicos se discuten en función de las particularidades ambientales, las materias primas disponibles en cada uno de los casos de estudio, y los contextos sociopolíticos de cada uno de los periodos. Los resultados demuestran una clara tendencia a sustituir la cal por arcillas en las argamasas del Clásico Terminal, lo cual lo atribuimos a la decadencia de técnicas constructivas causada por el deterioro sociopolítico de los sitios y posiblemente la deforestación extensiva. Se discute también el aprovisionamiento de materias primas para la fabricación de materiales constructivos y los problemas de la cronología establecida para los edificios de Palenque.
\end{abstract}

ABstract: This paper presents the results of petrographic and X-ray fluorescence analyses carried out in lime plaster samples from Calakmul and Palenque. Technological differences observed in the samples are though to represent different building traditions and these changes are discussed in terms of the environmental contexts, the availability of raw materials, and the socio-political contexts of each of the chronological periods. A clear tendency of replacing lime for clays in order to produce mud plasters was clearly documented in Terminal Classic materials. We think this is a consequence of the breakdown in building traditions caused by the collapse of the sociopolitical structure of the sites, exacerbated perhaps by shortages of firewood. The selection of raw materials and the problems in the chronology of Palenque's buildings is also discussed.

Palabras clave: cal, estuco, morteros, Palenque, Calakmul, caracterización de materiales, deforestación. Kfy words: lime, stucco, plaster, Palenque, Calakmul, materials caracterization, deforestation.

RECEPCIÓN: 9 de noviembre de 2007.

ACEPTACIÓN: 22 de octubre de 2008. 



\title{
UNA DE CAL POR LAS QUE VAN \\ DE ARENA: UN ESTUDIO DIACRÓNICO \\ DE LOS ESTUCOS DE CALAKMUL \\ Y PALENQUE
}

\author{
ISABEL VILLASEÑOR \\ Institute of Archaeology \\ University College, London \\ James Aimers \\ State University of New York at Geneseo
}

\section{Introducción}

En este artículo se presentan los resultados preliminares de composición y micromorfología obtenidos de materiales provenientes de Calakmul y Palenque. Estos dos sitios, que constituyeron entidades políticas independientes en época prehispánica, cuentan con recursos geológicos diferentes para la producción de cal, por lo que un análisis de sus materiales constructivos da cuenta de dos casos de estudio distintos. Sin embargo, estos sitios tuvieron también similitudes y puntos de contacto, ya que vivieron su apogeo constructivo en el Clásico Tardío —aunque la preeminencia de Calakmul se manifiesta desde el periodo Preclásico-, después de lo cual sufrieron una crisis sociopolítica y demográfica que repercutió en los programas arquitectónicos. Por esta razón, la comparación de los materiales de Calakmul y Palenque cobra cierto sentido.

Se presenta una breve introducción de la producción de cal y un resumen sobre las fuentes que informan acerca de la producción y el uso de este material en la zona maya. Después se describen los programas de arquitectura pública de los sitios bajo estudio, ya que es en este ámbito donde se dio el uso de la cal a gran escala. Posteriormente se presentan los procedimientos de laboratorio y los resultados de los análisis de muestras arqueológicas, seguido de la discusión y las conclusiones.

La cal en Mesoamérica y las fuentes de información sobre su producción

La cal se produce mediante la calcinación de piedras calizas u otros materiales ricos en carbonato de calcio, como las conchas o los corales, con los que se 
obtiene el óxido de calcio o cal viva. Este material se mezcla después con agua, lo que resulta en la cal apagada, que a su vez se mezcla con material de carga o agregados para formar una pasta que se aplica o modela sobre las superficies arquitectónicas. Tras el secado, la cal endurece y reacciona con el dióxido de carbono del aire para formar otra vez carbonato de calcio (Boynton, 1980). Los materiales de cal son, por lo tanto, una especie de piedra caliza sintética.

"Estuco" ha sido el término generalmente empleado en la arqueología mesoamericana para los materiales de cal empleados en la arquitectura. A pesar de que este término tiene otras acepciones en otros contextos académicos, en este artículo utilizamos este vocablo como un genérico debido a su uso generalizado.

La cal fue empleada como material constructivo en la gran mayoría de las culturas mesoamericanas. Sin embargo, a pesar de ser un material fundamental en la arquitectura prehispánica y de jugar un papel primordial en el desarrollo de estas civilizaciones, la investigación en la arqueología mesoamericana ha mostrado poco interés en estos materiales. No obstante, la concordancia de la información derivada de las fuentes históricas, las investigaciones etnográficas, así como los ejemplos donde se han documentado zonas de producción de cal en el registro arqueológico, confirman el sofisticado conocimiento que los mayas y otras culturas mesoamericanas tuvieron de este material y que continúa siendo una práctica viviente.

Los datos históricos y las fuentes etnográficas nos aportan información acerca de la producción de cal desde el siglo xvı, con base en lo cual se pueden realizar inferencias y analogías para el entendimiento del uso de este material en épocas prehispánicas. En el siglo xvı, fray Diego de Landa describió en su Relación de las Cosas de Yucatán (Tozzer, 1966: 176), la abundancia de la piedra caliza y del sac cab (sascab o saskab') en Yucatán, una tierra blanca que se utilizaba como material de carga para la fabricación de materiales de cal y que se sigue utilizando hasta la fecha de igual manera. Landa también describió el uso de sustancias orgánicas extraídas de cortezas de árboles, las cuales se mezclaban con la cal para mejorar las propiedades de los pisos y aplanados (Tozzer, 1966: 176).

Hernando Ruiz de Alarcón (1629) describió el quemado de la cal en comunidades nahuas de las tierras altas de México, en donde se conjuraba al viento y al fuego para que naciera la mujer blanca, yztac cihuatl o iztaccihuatl, la personificación de la cal. Estas prácticas coinciden perfectamente con las descripciones de Schreiner $(2002,2003)$ en contextos mayas actuales, como se explica más adelante.

Los primeros estudios etnográficos que documentaron la producción de cal en comunidades mayas contemporáneas fueron llevados a cabo por el Instituto Carnegie de Washington. Morris et al. (1931) documentaron detalladamente la producción de cal efectuada por maestros mayas para los trabajos de restauración en Chichén Itzá. Para esta producción se construyó una pira de troncos de madera formando un cilindro de 5.5 metros de diámetro y dos metros de altura, 
arriba de la cual se colocaron las piedras calizas para ser calcinadas (véase figura 1). Redfield y Villa (1934) documentaron el mismo procedimiento en Chan Kom, aunque con una pira más pequeña.

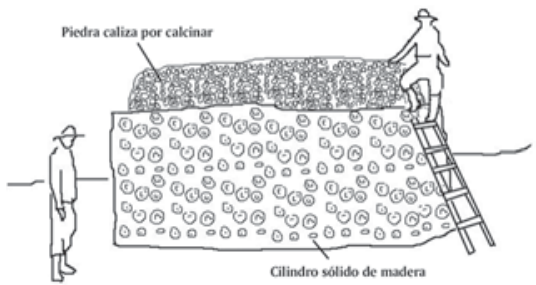

FIgURA 1. QUEmA DE CAL en la zona maya mediante piras al aire libre (caleras) Basado en Morris et al., 1931

Schreiner (2002), Russell y Dahlin (2007) han llevado a cabo también estudios etnográficos y experimentales de quemado de la cal con técnicas tradicionales mayas, y documentaron la misma técnica de quemado que los autores antes mencionados. Los investigadores analizan las implicaciones laborales y ambientales, así como los restos materiales dejados en la zona de producción. Schreiner (2002) describe siete diferentes variantes de construcción de piras de madera a cielo abierto, es decir, sin ningún tipo de estructura permanente de quemado, tanto a nivel de piso como en pozos. En todos los casos se usan maderas con alto contenido de agua, principalmente Chacáh (Bursera simaruba), ya que de esta forma se produce un quemado lento y controlado que promueve la mejor calcinación de la piedra caliza.

Esta técnica de quemado es relativamente ineficiente desde el punto de vista de la conservación del calor, ya que se requieren grandes cantidades de madera en comparación con el quemado en hornos, técnica que fue introducida por los españoles en el siglo xvı (Schreiner, 2002: 44). En la época prehispánica, las grandes cantidades de madera requeridas para la calcinación de la piedra resultaban seguramente en altas demandas laborales, ya que no existían animales de tiro para el transporte de materiales ni tampoco herramientas de metal para la tala de árboles. Por estas razones, la producción de cal ha sido considerada por varios autores como una de las actividades más demandantes de fuerza laboral en la industria de la construcción prehispánica (Barba y Córdoba Frunz, 1999; Abrams, 1994: 32; Abrams y Freter, 1996: 427). 
Se sabe también que la producción de cal en la zona maya se concibe como un nacimiento o transformación, en donde nace la mujer blanca o Sak Chu'pal, el equivalente maya de Iztaccíhuatl. De igual forma, la pira donde se calcina la piedra caliza se concibe como un útero, por lo que la cal y su producción están asociados con la feminidad, la pureza y la fertilidad (Schreiner, 2002: 104-117, 2003), simbología que coincide con los relatos del siglo xvi mencionados anteriormente. Aunque no lo sabemos por cierto, podemos pensar que este simbolismo existía desde la época prehispánica y que se extendía al uso de la cal en la arquitectura, por lo que las renovaciones de estructuras por medio de la aplicación de capas de repello y lechadas de cal pudieron haber conllevado una connotación de renacimiento o renovación relacionada a la simbología de la cal.

La investigación etnográfica también arroja luz acerca de la organización social de la producción de este material, la cual es llevada a cabo sólo por los hombres, mientras que a las mujeres se les prohíbe participar en la producción e incluso acercarse al área de quemado. Se sabe también que en las comunidades mayas actuales, la gran mayoría de los hombres están familiarizados con la cal y su producción (Schreiner, 2002: 100), lo que nos habla de la importancia de la cal en esta cultura.

La cal se usó de manera abundante en las tierras bajas mayas, debido en parte a que la península de Yucatán está constituida por una gran plataforma de calizas formada durante el Cenozoico (Espinosa et al., 1996). A pesar de la fácil obtención de la piedra caliza, la cal se usó de manera diferencial en los distintos niveles socioeconómicos de los sitios mayas; basta comparar la arquitectura pública monumental de mampostería que contiene grandes cantidades de cal, con los ejemplos de arquitectura doméstica en donde prevaleció la arquitectura de tierra con lechadas de cal. El uso preferencial de la cal se debe probablemente a la alta inversión laboral que este material demanda, lo que con toda probabilidad causaba que la cal en la arquitectura fuera considerada como un material de prestigio.

Sin embargo, la producción y el uso a gran escala de la cal en la arquitectura no debe confundirse con el consumo en el ámbito doméstico, en donde su empleo para el reblandecimiento del maíz o nixtamalización seguramente permeaba todos los estratos de la sociedad, como todavía se puede ver en comunidades indígenas. Este proceso, que consiste en remojar el maíz en agua de cal o algún otro medio alcalino como la ceniza, reblandece el pericarpio del grano y aumenta considerablemente el valor nutricional del maíz al aumentar el contenido en aminoácidos y calcio (Katz et al., 1974).

A pesar de la gran cantidad de cal que fue producida en épocas prehispánicas para la construcción de templos y diversas estructuras monumentales, son pocos los ejemplos en donde se ha detectado la producción de cal en el registro arqueológico (Abrams, 1996; Abrams y Freter, 1996; Castanzo, 2003; Castanzo y Anderson, 2004; Grove, 1987; Grove y Guillen, 1987; Russell, 2008). Esto se debe muy probablemente a que la cal en la época prehispánica también se producía a cielo abierto, sin ninguna estructura permanente de quemado. De ser así, los 
restos en el registro arqueológicos habrían sido sólo una fina capa de carbonatos y cenizas, lo que es difícil de detectar en las excavaciones. Por otra parte, es posible que la falta de detección de zonas de producción de cal se deba a la carencia de excavaciones extensivas en las zonas periféricas de los sitios, ya que éstas eran probablemente las zonas de producción debido a la abundancia de recursos forestales que podían ser aprovechados como combustibles.

Existen algunos estudios de caracterización de materiales de cal en la arqueología maya. Los primeros estudios fueron llevados a cabo por Littman $(1957,1958$, $1959,1959 b, 1960,1960 b, 1962,1966,1967$ y 1990) y recientemente por Hansen (2000), Hansen et al. (1995, 1997), Villegas et al. (1995), Ledezma y Vázquez (2002), García-Solís et al. (2006) y Goodall et al. (2007). También existen intentos por caracterizar las sustancias orgánicas contenidas en los estucos (Magaloni et al., 1992, Magaloni, 1995), e incluso trabajos experimentales para evaluar las características de los estucos cuando estas sustancias son empleadas como aditivos (Benavides, 2006).

\section{Estudios de caso: Calakmul y Palenque}

Tal como se describió anteriormente, la cal es un material con una alta demanda de recursos materiales y laborales. Por esta razón creemos que estos materiales deben analizarse de manera conjunta con los programas arquitectónicos a gran escala y con el momento sociopolítico que se vivía en cada periodo, ya que la inversión de energía en arquitectura pública está directamente relacionada con el desarrollo sociopolítico de las sociedades y con el estatus de las personas a cargo de las obras (Trigger, 1990). Por esta razón se presenta un breve resumen de los programas de arquitectura pública de Calakmul y Palenque y un breve esbozo de su historia política.

\section{Calakmul}

Calakmul es uno de los sitios mayas más grandes, aunque las excavaciones arqueológicas efectuadas en el sitio son relativamente recientes. El sitio se encuentra en el estado de Campeche, $30 \mathrm{~km}$ al norte de la frontera de Guatemala. Fue reportado por primera vez en 1931 por Cyrus L. Lundell, y explorado poco después por Sylvanus Morley del Instituto Carnegie de Washington. De 1982 a 1994 la Universidad de Campeche llevó a cabo un proyecto de excavación y mapeo bajo la dirección de William Folan. El INAH reinició los trabajos de investigación en 1993 bajo la dirección del arqueólogo Ramón Carrasco Vargas, los cuales continúan hasta la fecha.

El centro monumental de Calakmul fue construido sobre un domo natural de calizas, el cual es circundado por una franja de bajos, aunque también existen bajos y aguadas dentro del domo. La arquitectura monumental de Calakmul desde el 
Preclásico Medio demuestra la complejidad socio-cultural de este sitio desde épocas muy tempranas, así como la capacidad de organización de la élite gobernante, lo que es común con otros sitios de las tierras bajas centrales, tales como Nakbé, El Mirador y Uaxactún (Carrasco Vargas, 2000). Durante el periodo Preclásico se construyó la Estructura II y se configuró la acrópolis o Gran Plaza que se observa hasta nuestros días. Durante el Clásico Tardío, Calakmul siguió viviendo una época de esplendor y un crecimiento poblacional, y se construyeron las estructuras más voluminosas en la historia del sitio (Carrasco Vargas, 2000).

En contraste con otros sitios del Petén, Calakmul no fue abandonado a finales del Preclásico, sino que continuó ocupado durante todo el Clásico, aunque el entendimiento del periodo Clásico Temprano es todavía muy fragmentario (Carrasco Vargas, 2000). Sin embargo, algo que es claro, es que en el siglo vi d.C. Calakmul era ya un estado en expansión, como lo demuestran las inscripciones encontradas en varios sitios de las tierras bajas (Martin y Grube, 2000: 100-115), así como los importantes programas de arquitectura pública monumental y la elaborada ornamentación en sus tumbas (véase Carrasco 1996, 1999, 2000; Carrasco et al., 1999; Rodríguez Campero, 2008; Folan et al., 1995; García Moreno y Granados, 2000). Durante el periodo Clásico, Calakmul formó alianzas con otros sitios de las tierras bajas, principalmente con Caracol, para convertirse en el rival más importante de Tikal (Martin y Grube, 2000: 104), lo que afectó de manera determinante la vida política de las tierras bajas mayas durante el Clásico.

Calakmul y sus aliados vencieron a Tikal en 562 d.C., pero el apogeo constructivo de Calakmul se vivió durante el reino de Yuknoom Ch'een II (636-686 d.C.). A pesar de que Calakmul fue derrotado finalmente por Tikal en 695 d.C., Calakmul continuó con un extenso programa arquitectónico durante el reinado de Yuknoom Took' K'awiil de 702 a 731 d.C. (Martin y Grube, 2000). Sin embargo, poco después de esto Calakmul sufrió una disminución drástica en las actividades constructivas (Braswell et al., 2004).

Los estudios de población proponen que Calakmul alcanzó más de 50000 individuos, y posiblemente 200000 si se consideran sus sitios secundarios. A pesar de ello, Calakmul sufrió una crisis poblacional durante el Clásico Terminal, momento en que perdió probablemente el $90 \%$ de su población. En esta etapa ocurrieron cambios sociales y políticos importantes, y hay evidencia de que algunos edificios sagrados, como la Estructura II, fueron usados con fines residenciales y administrativos (Braswell et al., 2004). La última mención de un gobernante, Aj Took, ocurrió en 899 o 909 d.C. como parte de las celebraciones de un ciclo K'atun (Martin y Grube, 2000: 115).

También hay estudios que sugieren una disminución en la precipitación pluvial durante el Clásico Terminal (Braswell et al., 2004:190; Gunn et al., 2002b), e incluso quienes proponen que esta sequía se debió a una deforestación de carácter antrópico (Gunn et al., 2002a). Se cree también que la deforestación causó asimismo el secado de los cuerpos permanentes de agua, los cuales se transformaron en pantanos estacionales (Dunning, 2003). 


\section{Palenque}

Palenque se localiza en el área sudoccidental de las tierras bajas mayas, a los pies de la Sierra de Chiapas y los límites de la planicie aluvial de Tabasco.

Palenque fue reportado por primera vez en el siglo xvil, pero fue en el siglo XIX cuando se generó un gran interés europeo y cuando muchos viajeros visitaron el sitio, entre ellos John Stephens y Frederick Catherwood.

Palenque continuó siendo investigado de manera intensiva durante el siglo $\mathrm{xx}$, lo que ha enriquecido significativamente nuestro conocimiento sobre el sitio, pero fue con el comienzo de las mesas redondas de Palenque cuando se generó una nueva era de investigación en el sitio. La investigación epigráfica en Palenque ha sido particularmente fructífera para la arqueología maya, y ha arrojado valiosa información sobre la cosmogonía y sobre la historia dinástica del sitio. Uno de los descubrimientos más importantes de las investigaciones actuales es que Palenque y sus alrededores estaban mucho más densamente poblados de lo que se creía originalmente (Barnhart, 2000; Liendo Stuardo, 2000).

Aunque Palenque fue ocupado desde el Clásico Temprano, no fue sino hasta el Clásico Tardío cuando el sitio alcanzó su apogeo demográfico y político. K’inich Janahb' Pakal, quien reinó de 615 a 683 d.C., fue el pionero en las agendas constructivas con fines políticos. Pakal renovó el Palacio y encomendó el Templo Olvidado, que sería el modelo a seguir para edificios posteriores. Sin embargo, la obra cumbre de Pakal fue el Templo de las Inscripciones y su propia cripta funeraria contenida en el interior (Ruz-Lhuillier, 1973).

El hijo de Pakal, K'inich K'an B'ahlam II, lo sucedió en 684 d.C., y gobernó hasta 702 d.C. Con base en fuentes epigráficas, se cree que K'an B'ahlam continuó con los programas arquitectónicos de su padre y ordenó la construcción del Grupo de las Cruces, que incluye el Templo del Sol, el de la Cruz y el de la Cruz Foliada, que recuerda los arreglos triádicos de la arquitectura preclásica del Petén. Las actividades constructivas de periodos posteriores son de una escala menor, pero al parecer las renovaciones de la parte norte del Palacio fueron encomendadas por K'an B'ahlam II y su sucesor, K'inich K'an Joy Chitam II (Martin y Grube, 2000: 162-171).

El registro de los sucesores de K'an Joy Chitam II es menos claro, y la actividad constructiva es definitivamente menor. El último registro que se tiene de Palenque es de 799 d.C., y proviene de una vasija cerámica que documenta la subida al trono de Janab' Pakal III (Martin y Grube, 2000: 175). El colapso de Palenque fue uno de los más tempranos y dramáticos de la zona maya, y los análisis de la cerámica del sitio sugieren un colapso entre 800 y 830 d.C. (Rands, 1974).

\section{Análisis de materiales}

Se analizaron 21 muestras de Palenque y 25 de Calakmul, tanto de estucos como de materiales geológicos (piedras calizas y sascab). La mayoría de las muestras 
fueron analizadas por petrografía con la finalidad de documentar los materiales y características morfológicas de los estucos. Algunas muestras se procesaron también por medio de fluorescencia de rayos X (FRX) a fin de documentar la composición elemental de las mismas.

Las muestras fueron cortadas y adheridas en sección transversal a portaobjetos por medio de resina óptica, después de lo cual se cortaron con una sierra de diamantes y se desbastaron y pulieron hasta un grosor de $30 \mu \mathrm{m}$ usando abrasivos de óxido de aluminio. Las observaciones petrográficas se llevaron a cabo con un microscopio de luz polarizada Leica CMLP, en luz paralela y con nícoles cruzados, en aumentos entre $40 \mathrm{X}$ y $200 \mathrm{X}$, y se tomaron fotografías digitales con una cámara Coolpix.

Las muestras para fluorescencia de rayos X (FRX) se secaron a $100^{\circ} \mathrm{C}$ durante 24 horas y se trituraron en un molino de ágata de centrifugado. El polvo resultante se mezcló con $11 \%$ de cera analítica y se prepararon en pastillas comprimidas. Se utilizó un equipo Spectro XLab 2000 Pro de dispersión por longitud de onda para el análisis de las muestras. El oxígeno se combinó por estequiometría y los resultados se presentan como óxidos, a excepción del calcio y del magnesio que se reportan como carbonatos. Los resultados se normalizaron al $100 \%$ después de seleccionar los elementos significativos: $\mathrm{MgO}, \mathrm{Al}_{2} \mathrm{O}_{3}, \mathrm{SiO}_{2}, \mathrm{CaO}, \mathrm{TiO}_{2}$, $\mathrm{MnO}, \mathrm{Fe}_{2} \mathrm{O}_{3}$, $\mathrm{SrO}$ y $\mathrm{BaO}$.

Las muestras analizadas de Palenque provienen de templos construidos durante los reinados de K'inich Janahb' Pakal I (Templo del Conde), K'inich K'an Bahlam II (Grupo de las Cruces, Templo II, Grupo Norte), Kan Joy Chitam II (Casa A y D del Palacio), K'inich Kuk Bahlam II (Casa I del Palacio), así como de la fase Balunté (Grupo Murciélagos, Templo IV del Grupo Norte), la cual incluye desde el reinado de Kuk Bahlam II hasta la decadencia del sitio. Es importante mencionar que el fechamiento de los edificios de Palenque es difícil ya que no siempre es posible relacionar la evidencia epigráfica con la estratigrafía y las características arquitectónicas. Las fuentes consultadas para el fechamiento de las muestras incluyen estudios epigráficos y arqueológicos (Matthews 2006; Robertson, 1983b; Tovalín Ahumada y Ceja Manrique, 1993; Tovalín Ahumada y López Bravo, 2001), aunque la complejidad del fechamiento de los edificios de Palenque se discute más ampliamente en Marken y Lanham (2006).

En el caso de Calakmul, se analizaron muestras del Preclásico Medio/ Preclásico Medio Tardío (Subestructura IId y IIc1), Clásico Temprano (Acrópolis Chiik Naab', Complejo Residencial Chan Ch'ich y Estructura XIII), Clásico Tardío (Subestructura 1-I, Estructura GN-1 y subestructura XXa), así como del Clásico Terminal (Estructura Tok, edificio superior de la Estructura VI, y Estructura XX). Las muestras fueron fechadas por el equipo de trabajo dirigido por el arqueólogo Ramón Carrasco, principalmente con base en la relación de los edificios con tipologías cerámicas.

Un aspecto que se debe de tomar en cuenta es que los aplanados y pisos originales pudieron haber sido modificados mediante la aplicación de nuevas capas, y por lo tanto, los acabados visibles no necesariamente representan el mismo momento en que las estructuras fueron edificadas. 


\section{Resultados}

Fluorescencia de rayos $X(F R X)$

Palenque

En el caso de Palenque, los resultados de los análisis de fluorescencia de rayos $\mathrm{X}$ documentan alto contenido de calcio y de magnesio en las muestras, lo cual es un reflejo de la naturaleza calcárea y dolomítica, respectivamente, de las materias primas con las que se elaboraron los estucos. Sin embargo, las muestras Pa12 y Pa15 muestran alto contenido de calcio y bajo contenido de magnesio en comparación con el resto de las muestras.

Es posible también observar que las muestras $\mathrm{Pa} 17, \mathrm{~Pa} 20$ y en cierta medida Pa13, se distinguen claramente de las demás ya que tienen alto contenido de sílice, aluminio, hierro y titanio. Estas muestras provienen de las etapas más tardías: el Grupo de los Murciélagos, el Templo IV del Grupo Norte, y las modificaciones arquitectónicas de la casa D del Palacio (véase tabla 1).

Tавци 1. Análisis elementales de fluorescencia de rayos $\mathrm{X}$ de muestras de Palenque. Resultados normalizados de elementos seleccionados (reportados como porcentajes en peso y partes por millón). Los óxidos de calcio y magnesio se convirtieron a carbonatos antes de la normalización debido a la naturaleza de las muestras.

\begin{tabular}{|c|c|c|c|c|c|c|c|c|c|c|c|}
\hline & & & & & $\%(\mathrm{p}$ & eso) & & & ppm & peso) & \\
\hline Muestra & Tipo & Estructura & $\mathrm{MgCO}_{3}$ & $\mathrm{Al}_{2} \mathrm{O}_{3}$ & $\mathrm{SiO}_{2}$ & $\mathrm{CaCO}_{3}$ & $\mathrm{TiO}_{2}$ & $\mathrm{Fe}_{2} \mathrm{O}_{3}$ & SrO & $\mathrm{BaO}$ & Total \\
\hline Pa10 & Aplanado & Templo del Sol & 37.4 & 1.3 & 3.5 & 57.2 & 0 & 0.4 & 111 & 0 & 100 \\
\hline $\mathrm{Pa} 15$ & Piso & $\begin{array}{l}\text { Templo II, } \\
\text { Grupo Norte }\end{array}$ & 13.0 & 1.3 & 5.9 & 79.0 & 0 & 0.7 & 527 & 59 & 100 \\
\hline $\mathrm{Pa} 21$ & Aplanado & $\begin{array}{c}\text { Templo II, } \\
\text { Grupo Norte }\end{array}$ & 41.7 & 1.4 & 2.6 & 53.9 & 0 & 0.4 & 109 & 0 & 100 \\
\hline Pa8 & Piso & Casa D, Palacio & 34.7 & 1.1 & 5.4 & 58.2 & 0 & 0.4 & 281 & 113 & 100 \\
\hline $\mathrm{Pa} 12$ & Aplanado & Palacio Casa $\mathrm{A}$ & 9.1 & 1.1 & 4.3 & 84.9 & 0 & 0.5 & 348 & 58 & 100 \\
\hline Pa1 & Aplanado & Casa I, Palacio & 41.9 & 0.8 & 2.9 & 53.9 & 0 & 0.3 & 163 & 0 & 100 \\
\hline Pa13 & Aplanado & $\begin{array}{c}\text { Palacio Casa D } \\
\text { (muro divisorio) }\end{array}$ & 40.0 & 1.9 & 7.7 & 49.1 & 0.1 & 1.2 & 230 & 58 & 100 \\
\hline $\mathrm{Pa} 17$ & Piso & $\begin{array}{l}\text { Grupo de los } \\
\text { Murciélagos }\end{array}$ & 9.2 & 6.4 & 21.8 & 59.9 & 0.2 & 2.4 & 413 & 138 & 100 \\
\hline $\mathrm{Pa} 20$ & Aplanado & $\begin{array}{l}\text { Templo IV, } \\
\text { Grupo Norte }\end{array}$ & 16.9 & 4.9 & 19.9 & 55.9 & 0.2 & 2.1 & 200 & 67 & 100 \\
\hline Pa6 & Piedra & Templo del Conde & 37.7 & 0.5 & 2.4 & 59.2 & 0 & 0.2 & 163 & 54 & 100 \\
\hline $\mathrm{Pa} 4$ & Piedra & Templo XV & 40.6 & 1.0 & 2.2 & 56.1 & 0 & 0.1 & 108 & 0 & 100 \\
\hline $\mathrm{Pa} 5$ & Piedra & Juego de Pelota & 40.7 & 1.0 & 2.5 & 55.5 & 0 & 0.2 & 109 & 0 & 100 \\
\hline
\end{tabular}




\section{Calakmul}

En el caso de Calakmul la variación en composición química es menor que en Palenque. Sin embargo, se puede ver que las muestras Ca3, Ca22 y Ca23, del Clásico Terminal, forman un grupo distinto de las demás, ya que tienen bajas concentraciones de calcio, y alto contenido de hierro y silicio (véase tabla 2).

TABцa 2. Análisis elementales de fluorescencia de rayos $\mathrm{X}$ de muestras de Calakmul. Resultados normalizados de elementos seleccionados (reportados como porcentajes en peso y partes por millón). Los óxidos de calcio y magnesio se convirtieron a carbonatos antes de la normalización debido a la naturaleza de las muestras.

\begin{tabular}{|c|c|c|c|c|c|c|c|c|c|c|c|}
\hline & & & & & $\%(p$ & eso) & & & $\begin{array}{r}p p \\
\text { (pe }\end{array}$ & $\begin{array}{l}\text { so) } \\
\text { so) }\end{array}$ & \\
\hline Muestra & Tipo & Estructura & \begin{tabular}{|l|}
$\mathrm{MgCO}_{3}$ \\
\end{tabular} & $\mathrm{Al}_{2} \mathrm{O}_{3}$ & $\mathrm{SiO}_{2}$ & $\mathrm{CaCO}_{3}$ & $\mathrm{TiO}_{2}$ & $\mathrm{Fe}_{2} \mathrm{O}_{3}$ & $\mathrm{SrO}$ & $\mathrm{BaO}$ & Total \\
\hline Ca8 & Piso & $\begin{array}{c}\text { Subestructura } \\
\text { II-d }\end{array}$ & 1 & 1.1 & 13.6 & 83.2 & 0 & 0.3 & 1446 & 4526 & 100 \\
\hline Ca5 & Aplanado & $\begin{array}{c}\text { Subestructura } \\
\text { Ilc-1 }\end{array}$ & 1.8 & 1.7 & 13.6 & 82 & 0.1 & 0.5 & 1008 & 1386 & 100 \\
\hline Ca6 & Escultura & $\begin{array}{c}\text { Subestructura } \\
\text { llc-1 }\end{array}$ & 1 & 1.7 & 10.2 & 86.3 & 0.1 & 0.5 & 615 & 1169 & 100 \\
\hline Ca10 & Piso & $\begin{array}{l}\text { Estructura 1, } \\
\text { Subestructura } \\
\text { 1-4 Acrópolis } \\
\text { Chiik Naab' }\end{array}$ & 2.9 & 1.7 & 10.1 & 84.6 & 0.1 & 0.5 & 858 & 613 & 100 \\
\hline Ca15 & Piso & $\begin{array}{c}\text { Estructura 1, } \\
\text { Complejo } \\
\text { Chan Ch'ich }\end{array}$ & 1 & 1.1 & 10.3 & 87 & 0.1 & 0.3 & 735 & 1347 & 100 \\
\hline Ca14 & Piso & Estructura GN-1 & 1 & 1 & 13.5 & 83.9 & 0 & 0.3 & 501 & 2003 & 100 \\
\hline $\mathrm{Ca} 24$ & Aplanado & $\mathrm{XXa}$ & 1.1 & 0.9 & 9 & 88.4 & 0 & 0.3 & 787 & 908 & 100 \\
\hline $\mathrm{Ca} 3$ & Piso & Estructura Tok & 1.5 & 1.6 & 16.9 & 79 & 0 & 0.6 & 1225 & 2128 & 100 \\
\hline $\mathrm{Ca} 22$ & Piso & $\begin{array}{c}\text { Edificio } \\
\text { superior, } \\
\text { Estructura VI }\end{array}$ & 2.1 & 3.4 & 16.6 & 76.4 & 0.1 & 1.1 & 1568 & 1437 & 100 \\
\hline $\mathrm{Ca} 23$ & Aplanado & Estructura XX & 2 & 3 & 15.6 & 78.2 & 0.1 & 0.9 & 904 & 1485 & 100 \\
\hline $\mathrm{Ca} 20$ & Piedra & $\begin{array}{c}\text { Cantera } \\
\text { al Oeste } \\
\text { de Chiik Naab' }\end{array}$ & 0.9 & 0.8 & 3 & 94.9 & 0 & 0.2 & 1041 & 2082 & 100 \\
\hline $\mathrm{Ca} 21$ & Piedra & $\begin{array}{c}\text { Cantera al sur } \\
\text { de la pequeña } \\
\text { acrópolis }\end{array}$ & 0.8 & 0.9 & 2.9 & 95 & 0 & 0.3 & 463 & 289 & 100 \\
\hline Ca sascab & Sascab & $\begin{array}{c}\text { Biósfera } \\
\text { de Calakmul }\end{array}$ & 2.3 & 2.1 & 10.8 & 83.8 & 0.1 & 0.6 & 372 & 1548 & 100 \\
\hline
\end{tabular}




\section{Petrografia}

\section{Palenque}

Con base en las observaciones petrográficas, se pueden distinguir claramente tres grupos de texturas en los materiales de Palenque. El primer grupo presenta una matriz calcárea densa, de mezclado bueno y poros redondeados. El material de carga es exclusiva o predominantemente calcáreo de bordes subangulares o subredondeados. Otra característica de estas muestras es que se observan muchas capas y lechadas de cal, resultado de las renovaciones periódicas de los edificios. Este tipo de textura la presentan los materiales del Templo del Conde, el Grupo de las Cruces, y las casas A, D e I del Palacio: Pa1, Pa2, Pa3, Pa8, Pa11, Pa9, Pa10, Pa12, Pa14, Pa18 (figura 2).

El segundo grupo se caracteriza por una matriz arcillosa donde se distinguen múltiples fracturas. Las arcillas se observaron como zonas de partículas submicroscópicas de color café-rojizo tanto con luz paralela como con nícoles cruzados. También se observaron minerales opacos, muy probablemente óxidos de hierro, de manera concurrente con las arcillas. El material de carga es predominantemente cuarzo, en ocasiones policristalino, de bordes angulares o subangulares, y a veces acompañado de otros minerales accesorios como micas y feldespatos. Se pueden ver también nódulos de cal sin mezclar. Las muestras que presentan esta textura son más tardías que el primer grupo, e incluyen principalmente al Grupo de los Murciélagos, El Templo de la Calavera, y los edificios tardíos del Grupo Norte: Pa7, Pa13, Pa16, Pa19 y Pa21 (figura 2).

El tercer tipo de textura también presenta una matriz arcillosa rica en óxidos de hierro. Se observan también granos de cuarzo, aunque considerablemente más pequeños que en el grupo anterior (alrededor de $100 \mu \mathrm{m}$ ), por lo que probablemente estas inclusiones no fueron añadidas deliberadamente como material agregado, sino que formaban parte de los bancos arcillosos. Las muestras que presentan esta textura provienen también del Grupo de los Murciélagos, y de las modificaciones arquitectónicas del Grupo Norte: Pa17, Pa20 y la capa superior de Pa14 (figura 2).

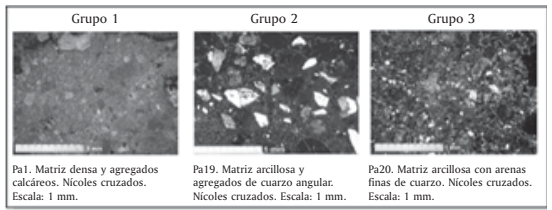

Figura 2. Grupos texturales de Palenque 


\section{Calakmul}

En el caso de Calakmul se pueden observar básicamente tres grupos de muestras. El primer grupo presenta minerales isotrópicos, muy probablemente sílice amorfo. Se puede ver una matriz densa, de poca porosidad, y con materiales calcáreos de bordes redondeados. En los poros se pueden ver claramente cristales aciculares. Las muestras de este grupo son principalmente del periodo Preclásico: $\mathrm{Ca} 5, \mathrm{Ca} 6, \mathrm{Ca} 7, \mathrm{Ca} 8$, Ca9 y Ca14.

El segundo grupo presenta las mismas características en la matriz y los agregados que el grupo anterior, aunque no se observan los cristales aciculares en los poros. Esta textura se distingue principalmente en el Clásico Tardío, así como en algunas muestras del Clásico Temprano: Ca4, Ca11, Ca12, Ca13 y Ca26.

El tercer grupo, al igual que en Palenque, presenta una mayor cantidad de arcillas en las mezclas, razón por la cual se observan múltiples fracturas en las matrices. Se encuentran fibras vegetales entre las grietas y el material de carga es calcáreo. Estas muestras son principalmente del Clásico Temprano y del Clásico Terminal: Ca1, Ca2, Ca3, Ca10, Ca15, Ca16, Ca17, Ca18, Ca22, Ca23 (véase figura 3).

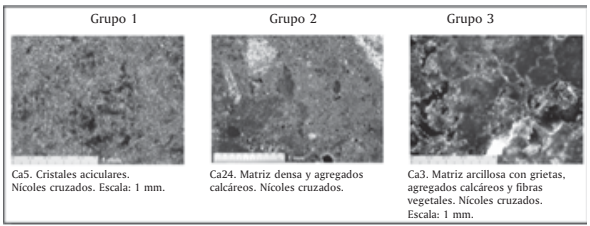

FigurA 3. Grupos texturales de Calakmul

\section{Discusión}

Los materiales constructivos muestran una naturaleza calcárea en la mayoría de los casos, reflejo de la larga tradición prehispánica del uso de la cal, y de la abundancia de las materias primas para su producción.

En el caso de Palenque hay muestras que presentan una gran cantidad de capas de estuco y lechada de cal, lo cual se atribuye al mantenimiento de los edificios. En el caso del Grupo de las Cruces, esto ha sido interpretado como evidencias de prácticas rituales de renovación de los templos (Villaseñor et al., en prensa).

Los cristales con hábitos aciculares observados en Palenque y las fases isotrópicas observadas tanto en materiales de Calakmul como de Palenque sugieren 
quizá el uso de cales hidráulicas mediante la incorporación de material volcánico. Es bien sabido que tanto los cristales aciculares como las fases isotrópicas son las características más fácilmente observables de las cales hidráulicas, las cuales han sido reportadas en materiales arqueológicos de otras zonas culturales (Charola y Enriques, 1999). Se sabe que los mayas emplearon ceniza y vidrio volcánico como materiales desgrasantes en cerámica (Shepard, 1954, 1964; Simmons y Brem, 1979; Jones, 1986; Ford y Glicken, 1987), mientras que los teotihuacanos emplearon el vidrio volcánico en estucos (Barba et al., 2006), por lo que es probable que los mayas también hubieran utilizado materiales volcánicos para la obtención de propiedades hidráulicas en sus argamasas. Esto continúa siendo investigado y se publicará más adelante en otro artículo.

Es claro que hay una tendencia a sustituir la cal por tierras y arcillas durante el Clásico Terminal, tanto en Calakmul como en Palenque, y en cierta medida también en el Clásico Temprano en Calakmul. Aunque no se identificaron los minerales arcillosos presentes en las muestras debido a que no se efectuó un análisis de composición mineralógica, la presencia de arcillas se confirmó mediante las observaciones petrográficas, así como con los resultados de fluorescencia de rayos $\mathrm{X}$, en donde se observó que el hierro covaría con el titanio, el aluminio, el silicio y el hierro, elementos presentes en la tierra y los minerales arcillosos.

En el caso de Palenque, es evidente que la técnica de manufactura de los estucos muestra poca variación antes del complejo cerámico Balunté (770-850 d.C.). Antes del 770 d.C. se observan estucos buenos y resistentes y con frecuentes renovaciones periódicas, lo que corresponde cronológicamente con los grandes programas arquitectónicos del centro cívico-ceremonial. Sin embargo, los materiales más tardíos, como son el Templo IV del Grupo Norte, el Grupo de los Murciélagos, y el Templo de la Calavera, muestran características completamente diferentes, en donde la cal se remplaza por arcillas y los agregados calcáreos por arenas de cuarzo.

Por medio de observaciones in situ en Palenque se pueden ver claramente modificaciones arquitectónicas que alteran la planta original de los edificios y que corresponden a las etapas más tardías del sitio (Cuevas García y González Cruz, 2007), en donde los materiales son también de carácter arcilloso. Algunos ejemplos de estas modificaciones arquitectónicas son los muros añadidos a los edificios del Grupo de la Cruz, la Casa D del Palacio, y el Grupo Norte (véase figura 4).

Aunque a simple vista es posible pensar que estas modificaciones arquitectónicas se llevaron a cabo en una etapa de ocupación posterior o de peregrinaje, los estudios petrográficos y de composición realizados en este estudio demuestran una clara similitud entre los materiales de edificios tardíos, como es el Grupo de los Murciélagos (Pa17), y los de los muros añadidos del Grupo Norte (Pa20), así como las observaciones in situ de los muros divisorios del Palacio y del Templo de la Cruz Foliada. Esto sugiere que los muros divisorios y otras modificaciones arquitectónicas fueron realizados en una etapa tardía del sitio y no en momentos de peregrinaje posterior al abandono. 

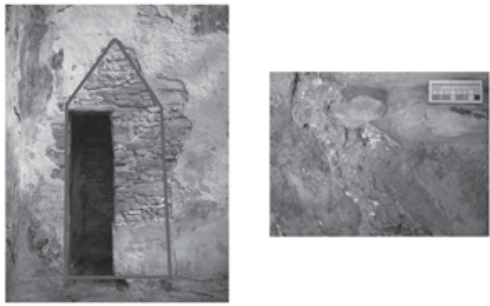

Figura 4. Modificaciones (muros añadidos) en la fachada principal del Templo de la Cruz Foliada (izq.). Detalle de materiales arcillosos con nódulos de cal sin mezclar (der.)

Por otra parte, la muestra Pa1, de la Casa I del Palacio, presenta problemas para su fechamiento. Aunque Robertson (1983a) asocia la construcción de la casa I con K'inich Kuk Bahlam II (764-783 d.C.), correspondiente al complejo cerámico Balunté, esta muestra guarda más parecido con los materiales más tempranos.

De manera similar al caso de Palenque, es evidente también el uso de arcillas y tierras en Calakmul durante el Clásico Terminal y hasta cierto punto también durante el Clásico Temprano. La observación del uso de aplanados de tierra ya ha sido reportada por Braswell et al. (2004) y Folan et al. (2001) en aplanados del Clásico Terminal en Calakmul, aunque no se había llevado a ningún estudio de caracterización de materiales.

Una explicación para el uso de las arcillas durante el Clásico Terminal, tanto en Calakmul como en Palenque, puede estar relacionada con la deforestación, ya que los materiales de tierra no son sometidos a un proceso de quemado, en contraste con los materiales de cal, y por lo tanto no requieren de combustible para su fabricación, por lo que habrían sido una buena alternativa en un paisaje deforestado. Es muy probable que la producción de cal en la época prehispánica se efectuara en piras abiertas, tal como se practica actualmente en comunidades mayas, la cual es una técnica de quemado que requiere de gran cantidad de madera como combustible. También es muy probable que los sitios se encontraran deforestados en las etapas más tardías, por lo que la madera se habría encontrado en zonas cada vez más distantes de la zona monumental, y el trabajo de transportación de madera era por lo tanto cada vez más demandante. Schreiner (2002: 71) describe que la fuerza laboral en la producción de cal aumenta de manera drástica cuando se incrementa la distancia de transportación de materiales, aún cuando se trata de distancias cortas. 
Por otra parte, es necesario también considerar que el rompimiento de las tradiciones constructivas observado en los materiales más tardíos puede ser resultado no sólo de la escasez de recursos forestales y de la alta demanda laboral, sino también de la crisis sociopolítica que afectaba a la mayoría de las tierras bajas durante el Clásico Terminal, lo que habría resultado en una incapacidad por parte de la élite político-administrativa para manejar la fuerza laboral que se requiere en la industria de la construcción. En este sentido cabe mencionar el caso de Teotihuacan (Barba et al., en prensa), en donde a pesar de que la fuente más cercana de piedra caliza se localizaba a más de $60 \mathrm{~km}$ de distancia, los teotihuacanos hicieron uso de la cal en sus monumentos, lo que demuestra que la transportación de materias primas no es un problema cuando una sociedad puede organizar la fuerza laboral.

Es importante mencionar que los materiales arcillosos se observaron también en Calakmul durante el Clásico Temprano, a excepción de los materiales de la acrópolis de Chiik Naab', los que presentan una textura más parecida a los materiales del Clásico Tardío. Se sabe que el Clásico Temprano fue una época de expansión para Calakmul, debido al número de estelas que lo mencionan en otros sitios (Folan et al., 1995: 326); sin embargo, es posible que este periodo también haya sufrido una deforestación considerable como consecuencia de los siglos de explotación y densificación durante el Preclásico. Es necesario por ello contar con datos paleoambientales para poder argumentar las razones de los cambios observados en los materiales constructivos, así como continuar con las excavaciones arqueológicas para entender de mejor manera el Clásico Temprano en Calakmul.

En el caso de Palenque, también es evidente el cambio en los materiales de carga (agregados), en donde los materiales calcáreos se sustituyen por cuarzo angular. El aumento en los contenidos de granos de cuarzo en Palenque ha sido descrito previamente por Villegas et al. (1995), aunque sus análisis son de materiales más tempranos y es por lo tanto difícil compararlos con los resultados de este estudio.

En cuanto a los contenidos de magnesio en las muestras de Palenque, la mayoría de las muestras corresponde con la geología del sitio, la cual está formada por dolomías (Littman, 1959b), es decir, piedras calizas con una proporción alta de magnesio. Sin embargo, las muestras Pa12 y Pa15, de la casa A del Palacio y del Grupo Norte respectivamente, tienen una proporción notablemente mayor de calcio, lo que indica que la cal se extraía de materiales calcíticos con baja proporción de magnesio. Esta falta de concordancia entre la geología del sitio y algunos estucos de Palenque ya ha sido descrita por Littman (1959b) y recientemente por Villaseñor y Price (2008), quienes sugieren el uso de cal de concha. La cal producida a partir de conchas marinas o de ríos es altamente calcítica, y su uso ha sido documentado etnográficamente en la zona maya por Baer y Merrifield (1971), Nations (1979) y Mackinnon y May (1990) para su uso en la preparación del maíz. En este sentido cabe mencionar las investigaciones que se están llevando a cabo 
por Cuevas García y Alvarado Ortega (2008), quienes describen la posible explotación prehispánica de conchas fósiles para actividades constructivas.

\section{Conclusiones}

Podemos ver que los resultados de los análisis de materiales forman patrones consistentes que corresponden con las grandes divisiones cronológicas establecidas por las tipologías cerámicas. No obstante, en algunos casos los análisis de materiales de cal pueden arrojar información adicional para refinar la cronología establecida por la cerámica y la epigrafía.

A pesar de las diferencias geológicas de los dos estudios de caso, y de que muy probablemente la producción de cal se producía con materiales calizos locales que abundaban en ambos sitios, tanto en Calakmul como en Palenque se puede ver una clara tendencia hacia la disminución en el uso de la cal y su remplazo por materiales arcillosos durante el Clásico Terminal, lo que corresponde cronológicamente con la disminución de los programas arquitectónicos a gran escala. En el caso de Calakmul esto también se observa en cierta medida durante el Clásico Temprano. Se cree que el abandono en el uso de la cal está relacionado con la crisis sociopolítica de los sitios y la incapacidad de organización de la fuerza laboral para las actividades constructivas, aunque también pudo haber estado relacionado con una deforestación extensiva, ya que los materiales arcillosos no requieren de quemado para su producción. Sin embargo, es necesario apoyar la segunda hipótesis con estudios paleoambientales.

En cuanto a los materiales de carga o agregados, es claro que los materiales calcáreos se sustituyen por cuarzo en las etapas tardías de Palenque, lo que indica la explotación de diferentes depósitos, probablemente provenientes de la planicie aluvial. En el caso de Calakmul los agregados calcáreos son usados en todos los periodos sin ningún cambio apreciable a través del tiempo, lo cual se debe muy probablemente a la abundancia de sascab en el sitio.

El posible uso de materiales volcánicos para la preparación de cales hidráulicas es un tema de interés en la arqueología maya desde el punto de vista de la tecnología constructiva y de explotación de los recursos naturales. A pesar de ello, se requieren más estudios de caracterización para entender el fenómeno con más claridad.

Los altos contenidos de magnesio en la mayoría de las muestras de Palenque son el reflejo de la litología dolomítica del sitio, a excepción de un par de muestras que son altamente calcíticas, lo que posiblemente esté relacionado con la explotación de conchas fósiles, como las reportadas por Cuevas García y Alvarado Ortega (2008).

Este estudio demuestra que los materiales de cal aportan información valiosa para el entendimiento de las tradiciones constructivas en la arqueología maya. La información derivada de análisis de materiales arqueológicos, cuando es usada en 
conjunto con las fuentes epigráficas, etnográficas y etnohistóricas, puede ser usada como punto de partida para discusiones relacionadas con el aprovisionamiento de recursos, la fuerza laboral empleada en la arquitectura, la cronología de los edificios, las condiciones paleoambientales, e incluso como un medio para adentrarnos a la rica simbología que los mayas conferían a los materiales y procesos tecnológicos.

El uso y la producción de cal en épocas antiguas fueron y continúan siendo aspectos primordiales para la cultura maya. Además de su abundante presencia en la arquitectura, la cal establece relaciones sociales específicas en su organización laboral, reviste un rico simbolismo y juega un papel fundamental en la subsistencia de estos pueblos con alto consumo de maíz. El análisis de materiales arqueológicos de cal, por lo tanto, requiere de una reflexión profunda acerca de los valores asociados a ellos y demanda, una vez más, que la arqueometría no pierda de vista la dimensión antropológica de la cultura material.

\section{Agradecimientos}

Deseamos expresar nuestro agradecimiento a la doctora Martha Cuevas por sus comentarios a este escrito, así como por sus interesantes ideas y discusiones sobre la arqueología de Palenque. También queremos agradecer al profesor Clifford Price, la doctora Elizabeth Graham y la doctora Ruth Siddall por su constante interés en este proyecto, así como por sus ideas y aportaciones. Debemos reconocer asimismo la labor de los técnicos de laboratorio, Simon Groom, Kevin Reeves y Philip Connolly, por su ayuda en la preparación y el análisis de las muestras.

Agradecemos igualmente al Consejo de Arqueología del Instituto Nacional de Antropología e Historia, por haber permitido el muestreo de materiales arqueológicos, y de manera especial damos las gracias a los arqueólogos de Calakmul: Ramón Carrasco Vargas — director del proyecto-, Omar Rodríguez Campero y Verónica Vázquez López. Nuestro agradecimiento también al personal administrativo y a los custodios de la zona arqueológica de Palenque, por permitir y ayudar en la toma de muestras.

Finalmente, al Consejo Nacional de Ciencia y Tecnología (CONACYT), al igual que a la Graduate School de UCL, por su apoyo económico.

\section{BIBLIOGRAFÍA}

Abrams, E. M.

1994 How the Maya Built their World: Energetics and Ancient Architecture. Austin, Texas: University of Texas Press.

1996 "The Evolution of Plaster Production and the Growth of the Copan Maya State", Arqueologia Mesoamericana: Homenaje a William T. Sanders, pp. 193208. vol. 2, México: INAH. 
Abrams, E. M. y A. C. Freter

1996 "A Late Classic Lime-plaster Kiln from the Maya Centre of Copán, Honduras", Antiquity 70: 422-428.

Barba, L. A., D. Barca, G. M. Crisci, L. Manzanilla, D. Miriello, A. Ortiz y A. Pecci 2006 "Characterization of Lime Plasters from the Central Patio of Teopancazco, Teotihuacan (México) by Optical Microscopy, SEM-EDS and ICP-MS Laser Ablation", presentación en póster en International Symposium of Archaeometry, Quebec City.

L. Manzanilla, A. Ortiz, D. Barca, G. M. Crisci, D. Miriello y A. Pecci "Provenance of the Limestone Used in Teotihuacan, México: A Methodological Approach", Archaeometry (en prensa, aceptado para publicación en mayo 2008).

y J. L. Córdoba Frunz

1999 "Estudios energéticos de la producción de cal en tiempos teotihuacanos y sus implicaciones", Latin American Antiquity 10 (2): 168-179.

Baer, P. y W. R. Merrifield

1971 Two Studies on the Lacandones of México. Norman: Summer Institute of Linguistics of the Univesity of Oklahoma.

Barnhart, E. L.

2000 The Palenque Mapping Project, 1998-2000. Final Report.

Benavides Jaidar, $\mathrm{Y}$.

2006 "Los extractos vegetales usados como aditivos en morteros de cal con fines de conservación". Tesis de licenciatura en Conservación de bienes muebles, INAH-ENCRM.

Boynton, R. S.

1980 Chemistry and Technology of Lime and Limestone, 2a. ed., Londres, Chichester: Ed. Wiley.

Braswell, G. E., J. D. Gunn, M. R. Domínguez-Carrasco, W. J. Folan, L. Fletcher, A. MoralesLópez y M. D. Glascok

2004 "Defining the Terminal Classic at Calakmul, Campeche", The Terminal Classic in the Maya Lowlands: Collapse, Transition, and Transformation, A. Demarest, P. M. Rice y D. S. Rice (eds.). Colorado: Colorado Universtiy Press.

Carrasco Vargas, $R$.

1996 "Calakmul, Campeche", Arqueología Mexicana III (18): 46-51.

1999 "Tumbas reales de Calakmul. Ritos funerarios y estructuras de poder", Arqueología Mexicana VII (40): 28-31.

2000 "El Cuchcabal de la Cabeza de Serpiente", Arqueología Mexicana VII (42): 12-21. 
et al.

1999 "A Dynastic Tomb from Campeche, México: New Evidence on Jaguar Paw, a Ruler of Calakmul", Latin American Antiquity 10 (1): 47-58.

Castanzo, R. A.

2003 Tepeaca Kiln Project, reporte presentado a FAMSI, disponible en: http://www. famsi.org/reports/02021/index.html

Castanzo, R. A. y H. H. Anderson

2004 "Formative Period Lime Kilns in Puebla, México", Mexicon XXVI: 86-90, Alemania: Verlag Anton Saurwein.

Cuevas García, M. y A. González Cruz

2007 "A flor de piel: la superficie de los edificios palencanos como fuente de información sobre los últimos momentos de ocupación de la ciudad", ponencia en el VIl Congreso Internacional de Mayistas, Mérida, 8-14 de julio 2007.

y J. Alvarado Ortega

2008 "El mar de la creación primordial: un escenario mítico y geológico de Palenque", ponencia en la VI Mesa Redonda de Palenque, Palenque, Chiapas, noviembre 2008.

Charola, A. E. y F. M. A. Enriques

1999 "Hydraulicity in Lime Mortars Revisited", Historic Mortars: Characteristics and Tests, RILEM-TC 167, International Workshop, Paysley, Escocia (RILEM publications).

Dunning, N. P.

2003. "Birth and Death of Waters: Environmental Change, Adaptation and Symbolism in the Southern Maya Lowlands", Espacios mayas: usos, representaciones, creencias, pp. 49-76, A. Breton, A. Monod Becquelin y M. H. Ruz (eds.). México: UNAM, Instituto de Investigaciones Filológicas, Centro de Estudios Mayas.

Espinosa, L., M. Ceron y Y. A. Sulub

1996. "Limestone Rocks of the Yucatán Peninsula. Description of the Lithology and Physical Properties Based on the Results of Exploration, Investigation and Laboratory Tests", International Journal of Rock Mechanics and Mining Sciences and Geomechanics Abstracts 35 (4): 410-411.

Ford, A. y W. I. Rose

1995 "Volcanic Ash in Ancient Maya Ceramics of the Limestone Lowlands: Implications for Prehistoric Volcanic Activity in the Guatemala Highlands", Journal of Volcanology and Geothermal Research 66 (1):149-162. 
Folan, W. J., J. Marcus, S. Pincemin, M. R. Domínguez-Carrasco, L. Fletcher y A. MoralesLópez

1995 "Calakmul: New Data from an Ancient Maya Capital in Campeche, Mexico", Latin American Antiquity 6 (4): 310-334.

J. D. Gunn y M. R. Domínguez-Carrasco

2001 "Triadic Temples, Central Plazas, and Dynastic Palaces: A Diachronic Analysis of the Royal Court Complex, Calakmul, Campeche, México", Royal Courts of the Ancient Maya, pp. 223-259, T. Inomata y S. Houston (eds.). Oxford: Westview.

García Moreno, R. y J. Granados

2000 "Tumbas reales de Calakmul", Arqueología Mexicana VII (42), pp. 28-33.

García-Solís, C. A., P. Quintana y E. Bautista-Zúñiga

2006 "La identificación de materiales arcillosos y pétreos utilizados en la manufactura del friso modelado en estuco de la Subll-C1 de Calakmul a través de análisis de difracción de rayos X", La ciencia de materiales y su impacto en la arqueología, pp. 237-251, M. Mendoza, J. A. Arenas, J. L. Ruvalcaba y V. Rodríguez (eds.). México, D. F.

Goodall, R. A., J. Hall, H. G. M. Edwards, R. J. Sharer, R. Viel y P. M. Fredericks

2007 "Raman Microprobe Analysis of Stucco Samples from the Buildings of Maya Classic Copan", Journal of Archaeological Science 34 (4): 666-673.

Grove, D. C.

1987 "Raw Materials and Sources", Ancient Chalcatzingo, pp. 376-386, D. C. Grove (ed.). Austin: University of Texas Press.

- y A. C. Guillén

1987 "The Excavations", Ancient Chalcatzingo, pp. 21-55, D. C. Grove (ed.). Austin: University of Texas Press.

Gunn, J., J. F. Foss, W. J. Folan, M. R. Domínguez-Carrasco y B. B. Faust

2002a "Bajo Sediments and the Hydraulic System of Calakmul, Campeche, México", Ancient Mesoamerica 13: 297-315.

R. T. Matheny y W. J. Folan

2002b "Climate Change Studies in the Maya Area", Ancient Mesoamerica 13 (1).

Hansen, E., R. D. Hansen y M. R. Derrick

1995 "Los análisis de los estucos y pinturas arquitectónicas de Nakbé: resultados preliminares de los estudios de los métodos y materiales de producción", ponencia en el VIII Simposio de Investigaciones Arqueológicas en Guatemala 1994 , Guatemala. 
C. Rodríguez-Navarro y R. D. Hansen

1997 "Incipient Maya Burnt Lime Technology: Characterization and Chronological Variations in Preclassic Plaster, Stucco and Mortar at Nakbe, Guatemala", ponencia en el Materials Research Society Symposium, Proceedings V: 207216. Boston, Masachusetts.

Hansen, R. D.

2000 "Ideología y arquitectura: poder y dinámicas culturales de los mayas del periodo Preclásico en las tierras bajas", ponencia en la Segunda Mesa Redonda de Palenque, Arquitectura e ideología de los antiguos mayas.

Jones, L. D.

1986 Lowland Maya Pottery: The Place of Petrological Analysis. Oxford: B.A.R., British Archaeological Reports, International Series (Publication 288).

Katz, S. H., M. L. Hediger y L. A. Valleroy

1974 "Traditional Maize Processing Techniques in the New World", Science 184 (4138): 765-773.

Ledezma, M. G. y J. Vázquez

2002 "Informe de resultados de los análisis practicados a los relieves estucados de la cripta funeraria del Templo de las Inscripciones, Palenque, Chiapas", informe no publicado, CNCPC-INAH.

Liendo Stuardo, R.

2000 "La población rural de Palenque", Arqueología Mexicana 2000: 34-37.

Littman, E. R.

1959 "Ancient Mesoamerican Mortars, Plasters and Stuccos. Las Flores, Tampico", American Antiquity 25 (1): 117-119.

1959 "Ancient Mesoamerican Mortars, Plasters and Stuccos. Palenque, Chiapas", American Antiquity 25 (2): 264-266.

1960 "Ancient Mesoamerican Mortars, Plasters and Stuccos. The Puuc Area", American Antiquity 25 (3): 407-412.

1960 "Ancient Mesoamerican Mortars, Plasters and Stuccos. The Use of Bark Extracts", American Antiquity 25 (4): 593-597.

1962 "Ancient Mesoamerican Mortars, Plasters and Stuccos. Floor Constructions at Uaxactun", American Antiquity 28 (1): 100-103.

1966 "The Classification and Analysis of Ancient Calcareous Materials", American Antiquity 31 (6): 875-878.

1967 "Patterns in Maya Floor Construction", American Antiquity 32 (4): 523-533. 
1990 Observations on the Floors of Platforms 5D-1 and 5D-4 (Group 5D-2), Tikal Report No. 14. Excavations in the Great Plaza, North Terrace and North Acropolis of Tikal. Filadelfia: University Museum, University of Pennsylvania.

1957 "Ancient Mesoamerican Mortars, Plasters and Stuccos. Comalcalco Part I", American Antiquity 23(2): 135-139.

1958 "Ancient Mesoamerican Mortars, Plasters and Stuccos. Comalcalco Part II", American Antiquity.

Mackinnon, J. J. y E. M. May

1990 "Small Scale Maya Lime Making in Belize, Ancient and Modern", Ancient Mesoamerica 1: 197-203.

Magaloni, D., T. Falcón, J. Cama, R. W. Siegel, R. Lee, R. Pancella, L. Baños y V. Castaño, 1992 "Electron Microscopy Studies of the Chronological Sequences of Teotihuacan Plaster Technique", Materials Research Society Symposium Proceedings 3: 997-1005.

R. Pancella, R. Fruh, J. Cañetas y V. Castaño

1995 "Studies on the Maya Mortars Technique", ponencia presentada en Materials Research Society Symposium, Proceedings, pp. 483-489. Pittsburg, Pensilvania.

Marken, D. B. y M. D. Lanham (eds.)

2006 Palenque: Recent Investigations at the Classic Maya Centre. Altamira Press, M.

D. Lanham.

Martin, S. y N. Grube

2000 Chronicle of the Maya Kings and Queens: Deciphering the Dynasties of the Ancient Maya. Londres: Thames and Hudson.

Morris, E. H, Charlot, J, y Morris, A. A

1931 The Temple of the Warrios at Chichen Itzá, Yucatán. Washington: Carnegie Institute of Washington.

Matthews, P.

2006 Who's Who in the Classic Maya World, reporte presentado a FAMSI, disponible en: http:/research.famsi.org/whos who/royal genealogy.php?site=Palenque

Nations, J. D.

1979 "Snail Shells and Maize Preparation: A Lacandon Maya Analogy", American Antiquity 44 (3): 568-571.

Rands, L. R.

1974 "A Chronological Framework for Palenque", Primera Mesa Redonda de Palenque, disponible en: http://www.mesoweb.com/pari/publications/RT01/ Chronological.pdf (consultado: octubre 2008). 
Redfield, R. y R. Villa

1934 Chan Kom: A Maya Village. Washington D. C.: Carnegie Institute of Washington (Publication 448).

Robertson, M. G.

1983a The Sculptures of Palenque. Volume III. The Late Buildings of the Palace. Princeton, Nueva Jersey: Princeton University Press.

1983b The Sculptures of Palenque. Volume IV. The Cross Group, the North Group, the Olvidado, and Other Pieces. Princeton, Nueva Jersey: Princeton University Press.

Rodríguez Campero, 0.

2008 La arquitectura Petén en Calakmul: una comparación regional, reporte presentado a FAMSI, disponible en: http://www.famsi.org/reports/02070es/index. html (consultado: octubre 2008).

Ruiz de Alarcón, $\mathrm{H}$.

1629 Treatise on the Heathen Superstitions that Today Live Among the Indians Native to this New Spain, 1629, trad. y ed. J. Richard Andrews y Ross Hassig, University of Oklahoma Press, The Civilization of the American Indian Series.

Russell, B. W. y B. H. Dahlin

2007 "Traditional Burnt-lime Production at Mayapán, México", Journal of Field Archaeology 32: 407-423.

Ruz-Lhuillier, A.

1973 El Templo de las Inscripciones, Palenque. México: INAH (Colección Científica).

Schreiner, T.

2002 Traditional Maya Lime Production: Environmental and Cultural Implications of a Native American Technology. Berkeley: University of California, Department of Architecture.

2003 "Aspectos rituales de la producción de cal en Mesoamérica: evidencias y perspectivas de las tierras bajas mayas", Memorias del Simposio de Investigaciones Arqueológicas en Guatemala, 2002, pp. 480-487. Guatemala: Museo Nacional de Arqueología y Etnología.

Shepard, A. O.

1954 Ceramics for the Archaeologists. Washington D. C.: Carnegie Institute of Washington (Publication 609).

1964 "Temper Identification: Technological Sherd-splitting or an Unanswered Challenge", American Antiquity 29 (4): 518-520. 
Simmons, M. P. y G. F. Brem

1979 "The Analysis and Distribution of Ash-tempered Pottery in the Lowland Maya Area", American Antiquity 44 (1): 79-91.

Tovalín Ahumada, A. y G. Ceja Manrique

1993 "Desarrollo arquitectónico del Grupo Norte de Palenque", ponencia en la Tercera Mesa Redonda de Palenque.

y R. López Bravo

2001 "Excavaciones en el norte del Palacio, Palenque", Revista Pueblos y Fronteras. México: PROIMMSE-UNAM, Instituto de Investigaciones Antropológicas.

Tozzer, A. M.

1966 Landa's Relación de las Cosas de Yucatán, Papers of the Peabody Museum of Archaeology and Ethnology, Harvard University, XVIII. Nueva York: Kraus.

Trigger, B. G.

1990 "Monumental Architecture: A Thermodynamic Explanation of Symbolic Behaviour", World Archaeology 22 (2).

Villaseñor, I. y C. A. Price

2008 "Technology and Decay of Magnesian Lime Plasters: The Sculptures of the Funerary Crypt of Palenque, México, Journal of Archaeological Science 35: 1030-1039.

M. Cuevas García y L. A. Barba

(en prensa) "Indicadores de actividad ritual en los templos del Grupo de las Cruces de Palenque, Chiapas", Memorias del Simposio de Investigaciones Arqueológicas de Guatemala 2008. Guatemala: Museo Nacional de Arqueología y Etnología.

Villegas, M., X. Vázquez, D. Ríos, L. Baños y D. Magaloni

1995 "Relative Dating of the Stucco Relieves at Palenque, Chiapas, Based on Variation of Material Preparation", Materials Research Society Symposium Proceedings 352: 469-481. 